\title{
Ultrafast optical modulation of Dirac electrons in gated single-layer graphene
}

\author{
Ikufumi Katayama $\odot,{ }^{1}$ Kei-ichi Inoue,${ }^{1}$ Yusuke Arashida, ${ }^{1}$ Yang Wu, ${ }^{2}$ Hyunsoo Yang $\odot,{ }^{2}$ Taiki Inoue, ${ }^{3}$ Shohei Chiashi $\odot,{ }^{3}$ \\ Shigeo Maruyama $\odot,{ }^{3,4}$ Tadaaki Nagao, ${ }^{5,6}$ Masahiro Kitajima, ${ }^{1,5}$ and Jun Takeda ${ }^{1}$ \\ ${ }^{1}$ Department of Physics, Graduate School of Engineering Science, Yokohama National University, Tokiwadai 79-5, \\ Hodogaya-ku, Yokohama 240-8501, Japan \\ ${ }^{2}$ Department of Electrical and Computer Engineering, National University of Singapore, 4 Engineering Drive 3, 117576, Singapore \\ ${ }^{3}$ Department of Mechanical Engineering, The University of Tokyo, Hongo 7-3-1, Bunkyo-ku, Tokyo 113-8656, Japan \\ ${ }^{4}$ Energy Nano Engineering Laboratory, National Institute of Advanced Industrial Science and Technology, \\ Namiki 1-2-1, Tsukuba, Ibaraki 305-8564, Japan \\ ${ }^{5}$ International Center for Materials Nanoarchitectonics (MANA), National Institute of Materials Science, \\ Namiki 1-1, Tsukuba, Ibaraki 305-0044, Japan \\ ${ }^{6}$ Department of Condensed Matter Physics, Hokkaido University, Sapporo, Hokkaido 060-0810, Japan
}

(Received 16 August 2018; revised manuscript received 24 March 2020; accepted 21 April 2020; published 5 June 2020)

\begin{abstract}
Fermi level dependence of ultrafast optical responses of single-layer graphene has been investigated using sub-10-fs pump-probe spectroscopy under bias voltages. We observe the ultrafast thermalization of photoexcited carriers, whose dynamics can be modulated via bias-induced change of the Fermi level. The relaxation time and the amplitude of the electronic response are maximized when the Fermi level reaches approximately half of the excitation photon energy. From the analysis of the pump-pulse-induced optical conductivity change, we find that the bias-induced blocking of the relaxation pathways and the pump-induced change of the electronic temperature and the Fermi level significantly contribute to the observed ultrafast optical modulation. The results demonstrate the controllability of the ultrafast optical responses in single-layer graphene, which could be useful for future ultrafast electro-optic graphene devices.
\end{abstract}

DOI: 10.1103/PhysRevB.101.245408

\section{INTRODUCTION}

Graphene shows many intriguing physical, optical, mechanical, and electric properties that are promising for future applications [1-4] and, thereby, its phonon and carrier dynamics have been studied intensively $[5,6]$. Previous attempts using ultrafast spectroscopy have revealed the importance of electron-electron and electron-lattice scatterings in ultrafast relaxation processes of photoexcited Dirac electrons along the nonparabolic electronic band [7-12]. The nonparabolic band structure also contributes to the field-driven nonlinear processes recently observed: in the efficiency of high-harmonic generation and ultrafast quantum interference of Dirac electrons, in which the electron scattering processes in femtosecond timescale have significant influence $[13,14]$.

The Fermi level of graphene is expected to affect the carrier dynamics through the change in carrier densities and distribution in the momentum space. Therefore, experimental studies in precise tuning of the Fermi level provide new insights for the underlying mechanism of ultrafast carrier dynamics in graphene [4,15-17]. In the present study, we perform sub-10-fs pump-probe spectroscopy on gated singlelayer graphene (grown by chemical vapor deposition), which provides systematic understanding of the ultrafast carriercarrier and carrier-phonon scatterings as a function of the electrically tuned Fermi level [4]. Our results reveal that the carrier relaxation, as well as the generation of coherent phonons, have strong dependence on the Fermi level, demonstrating a promising capability of controlling ultrafast optical response of graphene with the gate voltage.

\section{EXPERIMENT}

We used a sub-10-fs Ti:sapphire oscillator with an output power of $300 \mathrm{~mW}$, a repetition rate of $80 \mathrm{MHz}$, and laser spectrum ranging from $650 \mathrm{~nm}(1.91 \mathrm{eV})$ to $1050 \mathrm{~nm}(1.18 \mathrm{eV})$ (center wavelength of about $800 \mathrm{~nm}, 1.55 \mathrm{eV}$ ) for this work [18]. The laser output was attenuated and split into pump $(60 \mathrm{~mW})$ and probe $(5 \mathrm{~mW})$ beams with parallel polarization, which were then focused at a mutual angle of about $10^{\circ}$ on single-layer graphene with a silicon/ $\mathrm{SiO}_{2}$ substrate to perform a degenerate pump-probe experiment [19]. The spot size of the pump and probe pulses were approximately $20 \mu \mathrm{m}$ in diameter $\left(2 \times 10^{4} \mathrm{~W} / \mathrm{cm}^{2}\right)$. After spatially blocking the reflection of the pump, the reflection of the probe from the sample was fed into a photodiode with a preamplifier, and the pump-induced reflectance change was recorded and analyzed. The delay between pump and probe pulses was scanned by an optical shaker with a total delay range of $15 \mathrm{ps}$ at $20 \mathrm{~Hz}$. To apply a gate bias voltage to the single-layer graphene sample, we fabricated a gate electrode with ionic liquid as shown in Fig. 1(a) $[3,4]$. A transparent conductor of indium tin oxide (ITO) was used as a counter electrode, while the graphene sample itself acted as the other working electrode. The change of the sample resistance was simultaneously monitored by another pair of electrodes attached beside the graphene sample. 

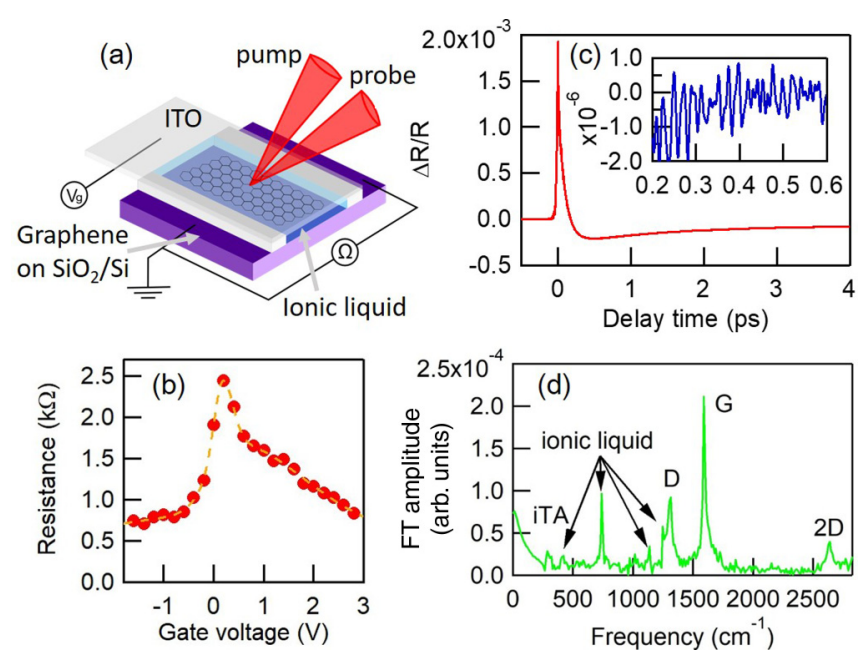

FIG. 1. (a) Schematic of the sample structure. Ionic liquid was sandwiched by single-layer graphene on $\mathrm{SiO}_{2} / \mathrm{Si}$ substrate and an indium tin oxide (ITO) coated glass. The resistance of the graphene under applied gate voltage was measured using a digital multimeter. Pump and probe beams were shone on the graphene through the ionic liquid, and the reflectivity change of the probe pulse was measured. (b) Observed resistivity of graphene under the gate voltage. (c) Typical reflectivity change of the graphene without gate voltage. Inset shows the oscillatory coherent phonon signals after subtracting the electronic responses. (d) Fourier transformed spectrum of the oscillatory components in (c). The iTA, D, G, and 2D (or $\mathrm{G}^{\prime}$ ) modes of graphene, as well as some vibrational modes of the ionic liquid are observed, as indicated by arrows.

The observed bias voltage dependence of the resistance is shown in Fig. 1(b), suggesting that the charge neutral point was achieved in this particular sample at $0.2 \mathrm{~V}$. Note that the bias voltage dependence of the resistance shows an asymmetric shape [20,21], indicating the different responses of the ionic liquid for electron and hole doping regimes [4]. The bias-dependent frequency shift of the G-mode phonon was used for the calibration of the Fermi level as discussed later. The ultrafast experiments were performed at intervals of $\sim 10$ min when we changed the experimental conditions such as the gate voltage, pump fluence, etc., ensuring thermal equilibrium of the graphene sample for each of the data points. We also confirmed duplicability of experimental results after a couple of months.

In Fig. 1(c), we show a typical example of the pump-probe signal of graphene obtained without a gate bias voltage. As shown in this figure, the pump pulse induces an instantaneous increase of the reflectivity, and the reflectivity decays with two components: the faster relaxation from the initially excited electronic states, and a subsequent slower heat transfer from photoexcited carriers to the lattice. According to the previous dynamical investigations of graphene, the faster decay $(\sim 100 \mathrm{fs})$ corresponds to the electronic thermalization due to electron-electron and electron-optical-phonon scatterings, while the slower component (a few ps) originates from the electron-acoustic-phonon scattering $[8,11,22,23]$. Note that the sign of the reflectance change is the opposite of that in the previous result, possibly because of the difference of the initial Fermi level of graphene or the experimental configuration

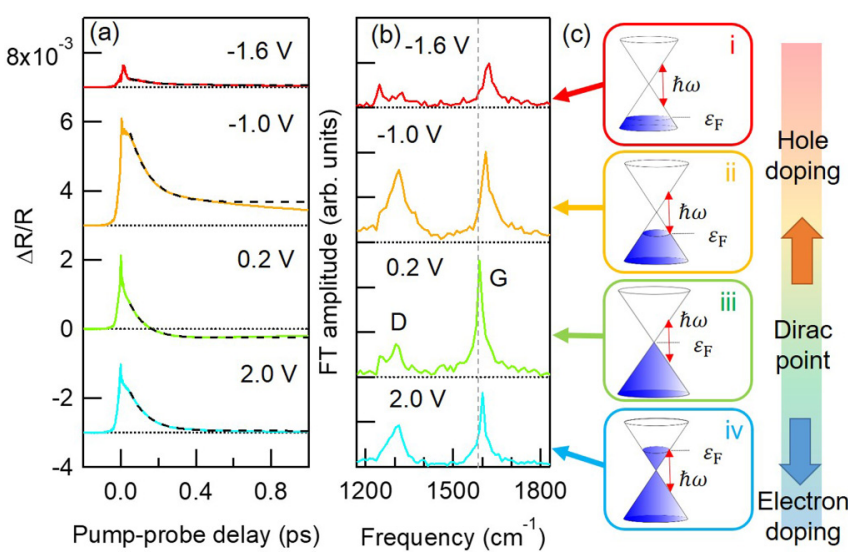

FIG. 2. (a) The reflectivity changes of single-layer graphene with different gate voltages. The dashed lines in (a) represent the single exponential fittings to extract the decay times of the fast component. Fourier transformed spectra of the oscillatory components are shown in (b). The dashed line in (b) is the peak position of the $\mathrm{G}$ mode when the Fermi level is at the Dirac point. (c) Schematic of the Fermi level at each bias voltage. Red arrows indicate the incident photon energy of the pump laser pulses resonant to the optical transition.

whether the substrate is opaque or not, and whether the change in the reflectivity or the transmittance was mainly observed [24]. Since the ultrafast modulation of the optical responses has been of interest in the present study, we mainly focus on the faster component in the following discussion. We fitted the data from 50 to $500 \mathrm{fs}$ using a single exponential function with a constant offset that represents the slow components. In addition to the ultrafast electronic response, we also observed high-frequency coherent phonon oscillations as shown in the inset of Fig. 1(c). The Fourier transformed (FT) spectrum of the oscillatory components, which was obtained by subtracting the electronic responses from the transient reflectivity, is depicted in Fig. 1(d) [25,26]. We can clearly observe the 2D (or $\mathrm{G}^{\prime}$ ) mode located at $2680 \mathrm{~cm}^{-1}(80 \mathrm{THz})$ together with the $\mathrm{G}$ and $\mathrm{D}$ modes, ensuring that the time resolution of our setup was below 10 fs. From the FT spectra, we can evaluate the center frequency and the amplitude of each coherent phonon.

\section{RESULTS AND DISCUSSIONS}

Figures 2(a) and 2(b) show the obtained transient reflectivity changes and FT amplitudes of coherent phonons in graphene with different gate voltages, respectively. We eliminated the effect of so-called coherent artifact near the time origin by smoothing the data with a 3 -fs Gaussian kernel $[27,28]$. Given the shifted neutral point evaluated from the results shown in Fig. 1(b), the graphene was either electron doped for the biases above $0.2 \mathrm{~V}$ or hole doped below $0.2 \mathrm{~V}$, as schematically shown in Fig. 2(c). The amplitude and decay rate of the electronic response have a strong dependence on the gate voltage. The amplitude reaches the maximum, while the relaxation time becomes slower at around $-1.0 \mathrm{~V}$. The slow negative transient reflectivity observed after $0.3 \mathrm{ps}$ around the charge neutral point $(\sim 0.2 \mathrm{~V})$ might be attributed to the reduction of the bandwidth of the $\pi$ band due to the thermal expansion of the lattice, which slightly enhances the 

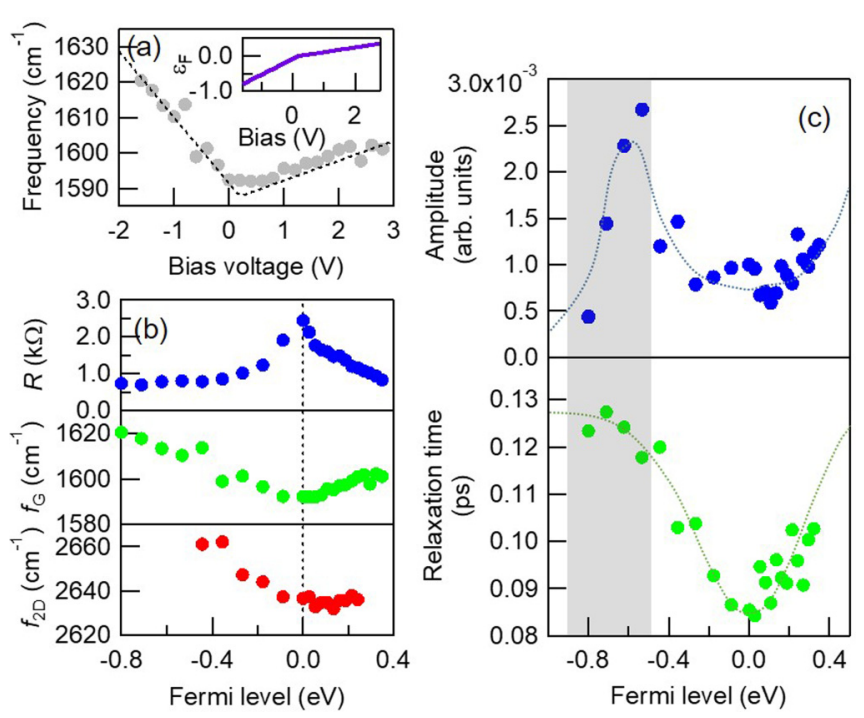

FIG. 3. (a) Gate-voltage dependence of the coherent G-mode phonon frequency. The dashed line shows an approximate linear relation between the applied bias voltage and the G-mode frequency. Note that the slope of these lines is different between negative and positive polarities. The inset shows the relation between the applied gate voltage and the Fermi level. (b) Fermi level dependence of the resistance and frequencies of $\mathrm{G}$ and $2 \mathrm{D}$ modes. (c) Amplitude and relaxation time for the faster component of the transient reflectivity changes observed at $t=10 \mathrm{fs}$ as a function of the Fermi level. The hatched area corresponds to the energy region where twice the Fermi level lies in the energy range of the laser spectrum. The dotted lines are the guides for the eyes.

absorption in the visible light region and then leads to the negative reflectivity. As is the case with the electronic responses, the coherent phonons of the G, D, and 2D modes also exhibit the bias dependence as shown in Fig. 2(b), which are consistent with the previous Raman scattering measurements [29]. The G-mode frequency varies with the gate voltage.

Figure 3(a) shows the G-mode phonon frequency as a function of the gate voltage. Since the frequency shift of the $\mathrm{G}$ mode, $\Delta \Omega_{\mathrm{G}}$, is directly proportional to the Fermi level $\varepsilon_{\mathrm{F}}$ as $\Delta \Omega_{\mathrm{G}}\left(\mathrm{cm}^{-1}\right)=\varepsilon_{\mathrm{F}} \times 42\left(\mathrm{~cm}^{-1} \mathrm{eV}^{-1}\right)$ [29], we can quantify the actual value of the Fermi level in our graphene sample using the frequency shift. Then, the gate-voltage dependence of the G-mode frequency, $f_{\mathrm{G}}(V)$, is given by the following empirical formula:

$$
f_{\mathrm{G}}(V)=f_{0}+\Delta \Omega_{\mathrm{G}}\left(\mathrm{cm}^{-1}\right)=f_{0}+a V .
$$

Here, $f_{0}=1589.8 \mathrm{~cm}^{-1}(47.6 \mathrm{THz})$ is the bare G-mode frequency [29,30], $a$ the proportionality constant, and $V$ the bias voltage. A dashed line in Fig. 3(a) represents the best fit with the parameter $a$, which is different between positive and negative gate voltage. Using Eq. (1), the Fermi level of the sample could be satisfactorily estimated as shown in the inset of Fig. 3(a). The estimation of the Fermi level from the G-mode phonon frequency minimizes the effect of the experimental inhomogeneity to determine the accurate Fermi level.

Figure 3(b) shows the replotted sample resistance and the G- and 2D-mode frequencies as a function of the Fermi level. As clearly shown in this figure, these curves are rather symmetric with respect to the Fermi level, indicating the validity of the analysis using G-mode frequency shift. The amplitude and the relaxation time of the faster component in the transient electronic response are shown in Fig. 3(c). The amplitude was evaluated near the time origin (pump-probe delay $t=10 \mathrm{fs}$ ), while the relaxation time was estimated from the fitting with a single exponential function. As highlighted by the hatched area in Fig. 3(c), a clear enhancement of the amplitude as well as the prolongation of the relaxation time is observed when the Fermi level is in the range between $\varepsilon_{\mathrm{F}}=-0.6$ and $\varepsilon_{\mathrm{F}}=-0.8 \mathrm{eV}$. In this area, $2\left|\varepsilon_{\mathrm{F}}\right|$ lies within the energy range in the laser spectrum $\left(1.18 \mathrm{eV} \leqslant 2\left|\varepsilon_{\mathrm{F}}\right| \leqslant\right.$ $1.91 \mathrm{eV})$. When $2\left|\varepsilon_{\mathrm{F}}\right|$ exceeds the photon energy of the excitation laser $\hbar \omega$ under the hole-doping condition [Fig. 2(c)-i], the optical transition is suppressed. If the relation $2\left|\varepsilon_{\mathrm{F}}\right| \sim \hbar \omega$ is satisfied [Fig. 2(c)-ii], the optical transition emerges as the reflectivity change with large amplitude. Simultaneously, the relaxation time for the electronic response might become longer, because the relaxation pathways are partially blocked by the occupied energy states $[7,20]$. In the case of $2\left|\varepsilon_{\mathrm{F}}\right| \ll \hbar \omega$ near the Dirac point [Fig. 2(c)-iii], the excited electrons easily relax to the lower-energy side, resulting in faster relaxation.

For further understanding of the underlying carrier dynamics, we consider the modulation of the Fermi level with photoexcitation [31]. The optical conductivity of graphene at a given electronic temperature $T$ and a Fermi level $\varepsilon_{\mathrm{F}}$ is expressed as [32-34]

$$
\sigma\left(T, \varepsilon_{\mathrm{F}}\right)=\frac{\pi e^{2}}{4 h}\left(\tanh \frac{\hbar \omega+2 \varepsilon_{\mathrm{F}}}{4 k_{\mathrm{B}} T}+\tanh \frac{\hbar \omega-2 \varepsilon_{\mathrm{F}}}{4 k_{\mathrm{B}} T}\right),
$$

where the conductivity exhibits a steplike dependence on the photon energy, $\hbar \omega$. When graphene is excited by pump pulses, nonequilibrium electron and hole distribution is generated, and subsequently, rapid thermalization takes place [11,12,35]. As a result, the electronic temperature and the Fermi level is modulated via the change of the distribution after the thermalization, leading to the variation of the optical conductivity [9,31]. The change of the conductivity,

$$
\Delta \sigma\left(T, \varepsilon_{\mathrm{F}}\right)=\frac{\partial \sigma\left(T, \varepsilon_{\mathrm{F}}\right)}{\partial \varepsilon_{\mathrm{F}}} \Delta \varepsilon_{\mathrm{F}}+\frac{\partial \sigma\left(T, \varepsilon_{\mathrm{F}}\right)}{\partial T} \Delta T,
$$

could then be calculated by estimating the relation between $\Delta \varepsilon_{\mathrm{F}}$ and $\Delta T$ when keeping the total carrier density constant in the Dirac cones [17].

As the optical conductivity depends on the photon energy $\hbar \omega$ as seen in the Eq. (2), we measure the probe wavelength dependence of the transient reflectivity. The results are summarized in Fig. 4. At first glance, the relaxation profile of the electronic response is almost independent of the probe wavelength except for the vicinity of the time origin, while the amplitude of the electronic response strongly depends on the probe wavelength. Therefore, hereafter, we focus on the Fermi level dependence of the amplitude. The normalized reflectivity change at $t=10 \mathrm{fs}$ is plotted as a function of the Fermi level for different probe wavelengths as shown in the top panel of Fig. 4(b). The calculated $\Delta \sigma\left(T, \varepsilon_{\mathrm{F}}\right)$ spectra with different probe wavelengths and temperatures are also depicted in the lower panel of Fig. 4(b). The longer the 

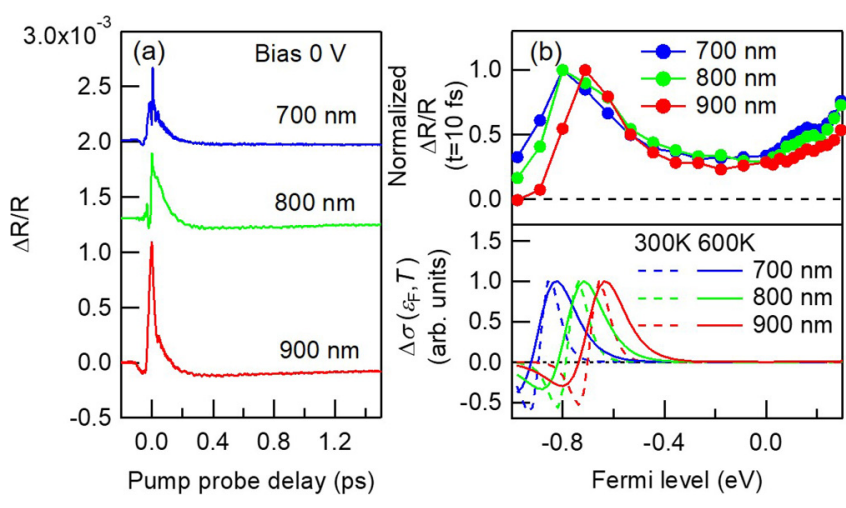

FIG. 4. (a) Transient reflectivity changes of single-layer graphene with different probe wavelengths, $700 \mathrm{~nm}(1.77 \mathrm{eV})$, $800 \mathrm{~nm}(1.55 \mathrm{eV})$, and $900 \mathrm{~nm}(1.38 \mathrm{eV})$, without bias voltage. The probe wavelength was separated by a band-pass filter with a bandwidth of $10 \mathrm{~nm}$. The vertical axis is shifted for clarity. (b) Fermi level dependence of the reflectivity change observed at given probe wavelengths at $t=10 \mathrm{fs}$. The lower panel shows the calculated result of $\Delta \sigma\left(T, \varepsilon_{\mathrm{F}}\right)$ described in the main text. Dashed lines correspond to the calculated results with the electronic temperature of $300 \mathrm{~K}$, while the solid lines are those of $600 \mathrm{~K}$.

probe wavelength, the higher the Fermi level for maximum signal amplitude. The observed result is in line with the calculated $\Delta \sigma\left(T, \varepsilon_{\mathrm{F}}\right)$ with temperature of $600 \mathrm{~K}$, implying the increased electronic temperature as indicated by the solid lines in Fig. 4(b). Discrepancy between the model and the experimental result observed at the low-bias region $(-0.4 \sim 0 \mathrm{eV})$ could be attributed to the nonthermal distribution of carriers in the Dirac band of graphene [36].

To clearly extract the relaxation processes from the nonthermal effects due to photoexcitation, we further plotted the Fermi level dependence of the transient reflectivity at longer delay times of $100 \mathrm{fs}$ and 2 ps. The result is summarized in Fig. 5. The difference between the model calculation and the experiment at the low-bias region is now almost removed in the longer delay-time regions, indicating that the electron distribution approaches the Fermi-Dirac distribution. In addition, the shift of the Fermi level for each probe wavelength becomes much clearer at the longer delay times, indicating that the model works well to evaluate the optically induced conductivity in graphene. Therefore, the wavelength dependence of pump-probe signals can be used to probe the electronic temperature of excited graphene.

The qualitative agreement of the calculation with the experiment unambiguously demonstrates that the pump-pulseinduced modulation of the electronic temperature and Fermi level significantly enhances the ultrafast optical responses in gated single-layer graphene, and the onset of the enhancement starts when the lowest edge of the laser spectrum is resonant to $2\left|\varepsilon_{\mathrm{F}}\right|$. Although the induced reflectivity change observed in this work is rather small, there has been a tremendous amount of works to enhance the modulation depth using specially designed graphene devices, and therefore, the obtained results

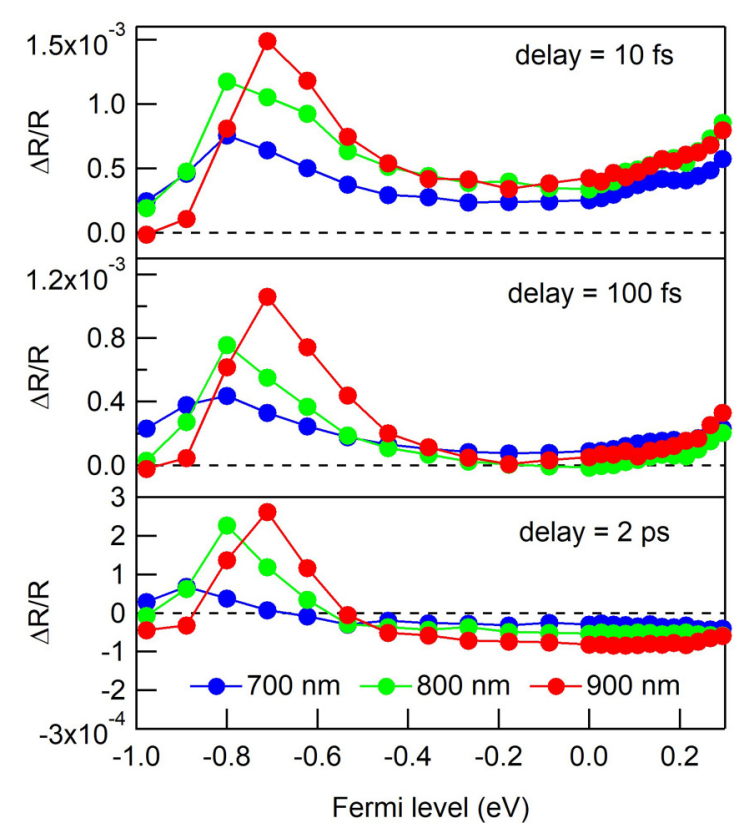

FIG. 5. Fermi level dependence of the reflectivity change observed at given probe wavelengths with longer delay times of $10 \mathrm{fs}$, $100 \mathrm{fs}$, and $2 \mathrm{ps}$.

provide the future outlook for the ultrafast optical modulation of graphene devices [37-40].

\section{CONCLUSION}

In conclusion, we have investigated the Fermi level dependence of ultrafast optical responses in gated single-layer graphene. We observe ultrafast electronic responses that originate from the electron thermalization and the electron-lattice thermalization, together with the coherent oscillations of the G, D, and 2D modes. The amplitude of the faster electronic response strongly depends on the Fermi level, and significantly enhances when twice the Fermi level reaches the lowest edge of the excitation laser spectrum. The relaxation time slows down at the Fermi level because of the blocking for relaxation pathways. The results could be understood by considering the change of the optical conductivity, which corresponds to the pump-pulse-induced modulation of the electronic temperature and the Fermi level. The observed ultrafast optical modulation of gated single-layer graphene gives additional perspective for the future applications of ultrafast two-dimensional devices.

\section{ACKNOWLEDGMENTS}

This work was supported by a Grant-in-Aid for Scientific Research (KAKENHI Grants No. 16H06364, No. 17H06124, No. $18 \mathrm{H} 03861$, No. $18 \mathrm{H} 04288$, and No. 19K22100) from the Ministry of Sports, Culture, Sciences and Technology. 
[1] A. H. Castro Neto, F. Guinea, N. M. R. Peres, K. S. Novoselov, and A. K. Geim, The electronic properties of graphene, Rev. Mod. Phys. 81, 109 (2009).

[2] A. K. Geim and K. S. Novoselov, The rise of graphene, Nat. Mater. 6, 183 (2007).

[3] E. O. Polat and C. Kocabas, Broadband optical modulators based on graphene supercapacitors, Nano Lett. 13, 5851 (2013).

[4] Y. Wu, C. La-o-vorakiat, X. Qiu, J. Liu, P. Deorani, K. Banerjee, J. Son, Y. Chen, E. E. Chia, and H. Yang, Graphene terahertz modulators by ionic liquid gating, Adv. Mater. 27, 1874 (2015).

[5] M. Liu, X. Yin, E. Ulin-Avila, B. Geng, T. Zentgraf, L. Ju, F. Wang, and X. Zhang, A graphene-based broadband optical modulator, Nature 474, 64 (2011).

[6] D. Sun, Z. K. Wu, C. Divin, X. Li, C. Berger, W. A. de Heer, P. N. First, and T. B. Norris, Ultrafast Relaxation of Excited Dirac Fermions in Epitaxial Graphene Using Optical Differential Transmission Spectroscopy, Phys. Rev. Lett. 101, 157402 (2008).

[7] S. Winnerl, M. Orlita, P. Plochocka, P. Kossacki, M. Potemski, T. Winzer, E. Malic, A. Knorr, M. Sprinkle, C. Berger, W. A. de Heer, H. Schneider, and M. Helm, Carrier Relaxation in Epitaxial Graphene Photoexcited Near the Dirac Point, Phys. Rev. Lett. 107, 237401 (2011).

[8] H. Choi, F. Borondics, D. A. Siegel, S. Y. Zhou, M. C. Martin, A. Lanzara, and R. A. Kaindl, Broadband electromagnetic response and ultrafast dynamics of few-layer epitaxial graphene, Appl. Phys. Lett. 94, 172102 (2009).

[9] M. Breusing, C. Ropers, and T. Elsaesser, Ultrafast Carrier Dynamics in Graphite, Phys. Rev. Lett. 102, 086809 (2009).

[10] C.-H. Park, F. Giustino, M. L. Cohen, and S. G. Louie, Electron-phonon interactions in graphene, bilayer graphene, and graphite, Nano Lett. 8, 4229 (2008).

[11] J. M. Dawlaty, S. Shivaraman, M. Chandrashekhar, F. Rana, and M. G. Spencer, Measurement of ultrafast carrier dynamics in epitaxial graphene, Appl. Phys. Lett. 92, 042116 (2008).

[12] M. Breusing, S. Kuehn, T. Winzer, E. Malić, F. Milde, N. Severin, J. P. Rabe, C. Ropers, A. Knorr, and T. Elsaesser, Ultrafast nonequilibrium carrier dynamics in a single graphene layer, Phys. Rev. B 83, 153410 (2011).

[13] N. Yoshikawa, T. Tamaya, and K. Tanaka, High-harmonic generation in graphene enhanced by elliptically polarized light excitation, Science 356, 736 (2017).

[14] T. Higuchi, C. Heide, K. Ullmann, H. B. Weber, and P. Hommelhoff, Light-field-driven currents in graphene, Nature 550, 224 (2017).

[15] I. Maeng, S. Lim, S. J. Chae, Y. H. Lee, H. Choi, and J. H. Son, Gate-controlled nonlinear conductivity of Dirac fermion in graphene field-effect transistors measured by terahertz timedomain spectroscopy, Nano Lett. 12, 551 (2012).

[16] F. Wang, Y. Zhang, C. Tian, C. Girit, A. Zettl, M. Crommie, and Y. R. Shen, Gate-variable optical transitions in graphene, Science 320, 206 (2008).

[17] A. Tomadin, S. M. Hornett, H. I. Wang, E. M. Alexeev, A. Candini, C. Coletti, D. Turchinovich, M. Kläui, M. Bonn, F. H. L. Koppens, E. Hendry, M. Polini, and K.-J. Tielrooij, The ultrafast dynamics and conductivity of photoexcited graphene at different Fermi energies, Sci. Adv. 4, eaar5313 (2018).
[18] J. Takeda, I. Katayama, K.-i. Shudo, and M. Kitajima, Coherent phonon and surface-enhanced Raman scattering dynamics in solids, J. Lumin. 152, 23 (2014).

[19] X. Chen, P. Zhao, R. Xiang, S. Kim, J. Cha, S. Chiashi, and S. Maruyama, Chemical vapor deposition growth of $5 \mathrm{~mm}$ hexagonal single-crystal graphene from ethanol, Carbon 94, 810 (2015).

[20] K. Maekawa, K. Yanagi, Y. Minami, M. Kitajima, I. Katayama, and J. Takeda, Bias-induced modulation of ultrafast carrier dynamics in metallic single-walled carbon nanotubes, Phys. Rev. B 97, 075435 (2018).

[21] K. Yanagi, R. Moriya, N. T. Cuong, M. Otani, and S. Okada, Charge Manipulation in Molecules Encapsulated Inside Single-Wall Carbon Nanotubes, Phys. Rev. Lett. 110, 086801 (2013).

[22] M. Wagner, Z. Fei, A. S. McLeod, A. S. Rodin, W. Bao, E. G. Iwinski, Z. Zhao, M. Goldflam, M. Liu, G. Dominguez, M. Thiemens, M. M. Fogler, A. H. Castro Neto, C. N. Lau, S. Amarie, F. Keilmann, and D. N. Basov, Ultrafast and nanoscale plasmonic phenomena in exfoliated graphene revealed by infrared pump-probe nanoscopy, Nano Lett. 14, 894 (2014).

[23] H. Wang, J. H. Strait, P. A. George, S. Shivaraman, V. B. Shields, M. Chandrashekhar, J. Hwang, F. Rana, M. G. Spencer, C. S. Ruiz-Vargas, and J. Park, Ultrafast relaxation dynamics of hot optical phonons in graphene, Appl. Phys. Lett. 96, 081917 (2010).

[24] B. A. Ruzicka, S. Wang, J. Liu, K.-P. Loh, J. Z. Wu, and H. Zhao, Spatially resolved pump-probe study of single-layer graphene produced by chemical vapor deposition [Invited], Opt. Mater. Express 2, 708 (2012).

[25] S. Koga, I. Katayama, S. Abe, H. Fukidome, M. Suemitsu, M. Kitajima, and J. Takeda, High-frequency coherent phonons in graphene on silicon, Appl. Phys. Express 4, 045101 (2011).

[26] I. Katayama, K. Sato, S. Koga, J. Takeda, S. Hishita, H. Fukidome, M. Suemitsu, and M. Kitajima, Coherent nanoscale optical-phonon wave packet in graphene layers, Phys. Rev. B 88, 245406 (2013).

[27] M. V. Lebedev, O. V. Misochko, T. Dekorsy, and N. Georgiev, On the nature of coherent artifact, J. Exp. Theor. Phys. 100, 272 (2005).

[28] S. L. Palfrey and T. F. Heinz, Coherent interactions in pumpprobe absorption measurements: the effect of phase gratings, J. Opt. Soc. Am. B 2, 674 (1985).

[29] C. F. Chen, C. H. Park, B. W. Boudouris, J. Horng, B. Geng, C. Girit, A. Zettl, M. F. Crommie, R. A. Segalman, S. G. Louie, and F. Wang, Controlling inelastic light scattering quantum pathways in graphene, Nature 471, 617 (2011).

[30] J. Yan, Y. Zhang, P. Kim, and A. Pinczuk, Electric Field Effect Tuning of Electron-Phonon Coupling in Graphene, Phys. Rev. Lett. 98, 166802 (2007).

[31] I. Gierz, J. C. Petersen, M. Mitrano, C. Cacho, I. C. E. Turcu, E. Springate, A. Stöhr, A. Köhler, U. Starke, and A. Cavalleri, Snapshots of non-equilibrium Dirac carrier distributions in graphene, Nat. Mater. 12, 1119 (2013).

[32] T. Stauber, N. M. R. Peres, and A. K. Geim, Optical conductivity of graphene in the visible region of the spectrum, Phys. Rev. B 78, 085432 (2008). 
[33] K. Ziegler, Minimal conductivity of graphene: Nonuniversal values from the Kubo formula, Phys. Rev. B 75, 233407 (2007).

[34] K. F. Mak, L. Ju, F. Wang, and T. F. Heinz, Optical spectroscopy of graphene: From the far infrared to the ultraviolet, Solid Stat. Commun. 152, 1341 (2012).

[35] I. Gierz, F. Calegari, S. Aeschlimann, M. Chavez Cervantes, C. Cacho, R. T. Chapman, E. Springate, S. Link, U. Starke, C. R. Ast, and A. Cavalleri, Tracking Primary Thermalization Events in Graphene with Photoemission at Extreme Time Scales, Phys. Rev. Lett. 115, 086803 (2015).

[36] L. A. Falkovsky, Optical properties of graphene, J. Phys.: Conf. Ser. 129, 012004 (2008).

[37] N. Kumada, P. Roulleau, B. Roche, M. Hashisaka, H. Hibino, I. Petkovic, and D. C. Glattli, Resonant Edge Magnetoplasmons and Their Decay in Graphene, Phys. Rev. Lett. 113, 266601 (2014).

[38] J. S. Park, A. Reina, R. Saito, J. Kong, G. Dresselhaus, and M. S. Dresselhaus, $\mathrm{G}^{\prime}$ band Raman spectra of single, double and triple layer graphene, Carbon 47, 1303 (2009).

[39] Q.-W. Sheng, M. Feng, W. Xin, H. Guo, T.-Y. Han, Y.-G. Li, Y.-G. Liu, F. Gao, F. Song, Z.-B. Liu, and J.-G. Tian, Tunable graphene saturable absorber with cross absorption modulation for mode-locking in fiber laser, Appl. Phys. Lett. 105, 041901 (2014).

[40] Y. Fan, C. Guo, Z. Zhu, W. Xu, F. Wu, X. Yuan, and S. Qin, Monolayer-graphene-based broadband and wide-angle perfect absorption structures in the near infrared, Sci. Rep. 8, 13709 (2018). 